\title{
Nasolacrimal duct laceration
}

\author{
Ariunbold Tumenjargal ${ }^{1 *}$ and Yen-Chang $\mathrm{Chu}^{2}$ \\ ${ }^{1}$ Orbita Eye Hospital, Department of Ophthalmology, Ach Medical University, Mongolia \\ ${ }^{2}$ Chang Gung Memorial Hospital, Chang Gung University, Linkou, Taiwan
}

The nasolacrimal duct laceration are involved in $30 \%$ of all lacrimal system injuries. Nasolacrimal duct lacerations can be the result of blunt trauma. Nasolacrimal ducts may also become obstructed by indirect trauma as an after effect of nasolacrimal laceration and facial and orbital bone fractures. The patient with nasolacrimal injuries may present with significant concurrent facial wounds, and multiple system injuries.

In this case report a 64-year-old woman is presented with deep facial laceration after a fall injury. The laceration extended from medial canthus region, anterior lacrimal crest to infraorbital region of midface (Figure A). In cases of traumatic nasolacrimal duct laceration was transected behind the minimally fractured anterior lacrimal crest (Figure B). The epidemiology, the most important principles of surgical repair of nasolacrimal duct, special techniques of reconstructive surgery. She received primary micro reconstructive repair with silicone tubing and the extended from medial nasal canthus region with local anesthesia. Two months after surgery, patient did not complain tearing and irrigation test was also patent. Lacrimal drainage laceration occurred more in upper part. (Figure C). However, nasolacrimal duct laceration is rare for the location within the bony canal. Identifying the distal cut end is usually unfamiliar for ophthalmologists.



Figure 1. (A) Shows the laceration extended from medial canthus region, anterior lacrimal crest to infraorbital region of midface (B) In cases of traumatic nasolacrimal duct laceration was transected behind the minimally fractured anterior lacrimal crest (C) Reveals the Lacrimal drainage laceration
Copyright: (C)2019 Tumenjargal A. This is an open-access article distributed under the terms of the Creative Commons Attribution License, which permits unrestricted use, distribution, and reproduction in any medium, provided the original author and source are credited.
${ }^{*}$ Correspondence to: Yen-Chang Chu, Chang Gung Memorial Hospital, Chang Gung University, Linkou, Taiwan, E-Mail: fredygeraud@gmail.com

Received: August 02, 2019; Accepted: September 06, 2019; Published: September 11, 2019 